\title{
Adaptive Flutter Suppression for a Fighter Wing via Recurrent Neural Networks over a Wide Transonic Range
}

\author{
Haojie Liu, Yonghui Zhao, and Haiyan Hu \\ State Key Laboratory of Mechanics and Control of Mechanical Structures, Nanjing University of Aeronautics and Astronautics, \\ Nanjing 210016, China
}

Correspondence should be addressed to Haiyan Hu; hhyae@nuaa.edu.cn

Received 23 July 2015; Revised 23 February 2016; Accepted 6 March 2016

Academic Editor: Saad A. Ahmed

Copyright (C) 2016 Haojie Liu et al. This is an open access article distributed under the Creative Commons Attribution License, which permits unrestricted use, distribution, and reproduction in any medium, provided the original work is properly cited.

The paper presents a digital adaptive controller of recurrent neural networks for the active flutter suppression of a wing structure over a wide transonic range. The basic idea behind the controller is as follows. At first, the parameters of recurrent neural networks, such as the number of neurons and the learning rate, are initially determined so as to suppress the flutter under a specific flight condition in the transonic regime. Then, the controller automatically adjusts itself for a new flight condition by updating the synaptic weights of networks online via the real-time recurrent learning algorithm. Hence, the controller is able to suppress the aeroelastic instability of the wing structure over a range of flight conditions in the transonic regime. To demonstrate the effectiveness and robustness of the controller, the aeroservoelastic model of a typical fighter wing with a tip missile was established and a singleinput/single-output controller was synthesized. Numerical simulations of the open/closed-loop aeroservoelastic simulations were made to demonstrate the efficacy of the adaptive controller with respect to the change of flight parameters in the transonic regime.

\section{Introduction}

Aeroelastic instability, induced by the interaction of unsteady aerodynamics and structural dynamics, is a disaster for any flight vehicles. Hence, recent years have witnessed a great number of studies, both numerical and experimental ones, on the design of aeroelastic control systems [1-5]. For example, Mukhopadhyay synthesized a flutter suppression controller for the active flexible wing model by using the linear quadratic Gaussian theory [1]. Furthermore, based on the unified linear quadratic Gaussian and minimax method, Mukhopadhyay proposed a flutter suppression controller, which was tested in the NASA Langley Transonic Dynamics Tunnel [2]. Then, Zhao proposed a flutter suppression controller for the aeroelastic system with input time delay in control loop via the $H_{\infty}$ control theory [3]. Afterwards, Moulin et al. studied the classic and robust controllers [4] and Zeng et al. studied an experimental model-based feedbackcontrol framework [5] for flutter suppression and gust load alleviation of the supersonic semispan transport wind-tunnel model. An active flutter controller should work well over a range of flight conditions since the flight parameters, such as flight velocity and Mach number, are always subject to variations during a flight. However, it is difficult to synthesize a controller for aeroelastic stabilization over a range of flight parameters via conventional control theory, such as linear quadratic Gaussian (LQG) control, because the aeroelastic system usually changes the stability with the variation of flight conditions.

Adaptive control methodology seems to be an appropriate solution because the parameters in such a control strategy are capable of adapting to the change of flight conditions. Different kinds of adaptive control methodology have been used to design the flutter suppression control laws. For example, Andrighettoni and Mantegazza designed an indirect adaptive control law for a wing model with a leading-edge and a trailing-edge control surface [6]. Then, Zhang and Singh proposed a new adaptive control system based on a modeling error compensation technique for an aeroelastic system with unstructured uncertainties [7]. Afterwards, Behal et al. proposed an adaptive strategy via employment of the full-state feedback for a wing section with multiple flaps 
[8]. Furthermore, Huang et al. proposed an adaptive output feedback-control law for the flutter suppression of a windtunnel model [9]. In addition, Mannarino and Mantegazza proposed a full-state immersion and invariance controller for active flutter suppression [10]. Very recently, Lee and Singh proposed an adaptive control system for an uncertain aeroelastic system by using leading-edge and trailing-edge control surfaces [11]. Compared with the vast body of applications of adaptive controls to aeroelastic systems, only a few adaptive control strategies involving the variation of flight conditions have been available. For example, Pak et al. [12] proposed a digital adaptive controller for aeroelastic stabilization under time-varying flight conditions. Their methodology combines Auto Regressive Moving Average (ARMA) model and online parameter estimation and a Riccati solver together to synthesize the online adaptive optimal control law. However, the performance of the controller depends on the flight paths as shown in the study. Then, Scott and Pado [13] designed and testified the flutter control systems based on static neural networks for the wind-tunnel model of Benchmark Active Controls Technology (BACT). They fixed the parameters of neural networks and used the gain scheduled control theory. Nevertheless, many control designs are essential for gain scheduled control to suppress aeroelastic instability over a range of flight conditions. Afterwards, Chen et al. [14] proposed the Linear Parameter-Varying (LPV) control theory so as to design a robust and implicit gain scheduled flutter controller in a transonic regime. In practice, the LPV aeroservoelastic (ASE) model for a range of flight conditions has to be established at first. Furthermore, Huang et al. [15] synthesized an adaptive flutter suppression system for the wing model of BACT in a transonic regime but had to initiate the adaptive controller by exciting the control surface with a white noise signal at each Mach number. As a matter of fact, it is difficult and dangerous for actuators of control surfaces to follow the command signals during real flight. Therefore, it is necessary to develop an adaptive flutter suppression system over a range of flight conditions.

In this study, the recurrent neural networks (RNNs) were used to design an adaptive flutter suppression system in a wide transonic range. Although RNNs have been applied to aeroelastic control in previous studies [16, 17], some issues are subject to further research, such as the application of the controller to flutter suppression in a transonic regime, and the robustness of the controller over a range of flight conditions. In the study, these issues were addressed for the adaptive flutter suppression over a wide range of flight conditions, including transonic regime, where the unsteady aerodynamic influence coefficient matrices were generated via an efficient methodology proposed by Chen et al. [18] for different flight parameters. After the ASE model was established, the parameters of RNNs based control system were initialized to suppress the aeroelastic instability at a Mach number and a dynamic pressure at first. Then, by updating the synaptic weights of networks online, the controller could automatically adjust itself to a new flight condition. As shown by numerical simulations, the adaptive controller for flutter suppression worked well over a wide range of flight conditions. As it is very difficult to establish an
TABLE 1: Mode frequencies of a typical fighter wing with a tip missile.

\begin{tabular}{lcc}
\hline Mode number & Frequency $(\mathrm{Hz})$ & Description \\
\hline 1 & 2.33 & Wing bending \\
2 & 6.39 & Wing torsional \\
3 & 16.99 & Wing bending \\
4 & 19.56 & Missile bending (in-plane) \\
5 & 25.83 & Missile bending \\
6 & 29.91 & Wing torsional \\
\hline
\end{tabular}

ASE model of high fidelity for designing a flutter controller, an important advantage of the RNNs based controller is no requirement for any information of the aeroelastic system except for the acceleration signals measured. The RNNs based controller is also a basis of the ongoing research on the suppression of Limit Cycle Oscillations (LCO).

The remainder of the paper is organized as follows. In Section 2, the ASE model of a typical fighter wing with a tip missile is established. Then, the main theory of RNNs based controller is presented in Section 3. In Section 4, numerical simulations are given to demonstrate the effectiveness and robustness of the proposed active flutter controller against changing flight parameters in a wide transonic regime. Finally, some conclusions are drawn in Section 5.

\section{Aeroservoelastic Model of a Typical Fighter Wing with a Tip Missile}

2.1. The Finite Element Model. In the study, the Finite Element Model (FEM), containing 486 nodes and 1973 elements, for a typical fighter wing with a tip missile was constructed by using MSC/NASTRAN. The first ten natural frequencies and the corresponding mode shapes were obtained via MSC/NASTRAN and used for open/closed-loop ASE analysis in Section 4. Figure 1 shows the first six mode shapes and Table 1 presents the corresponding natural frequencies. The first six modes are the first bending mode of the wing, the first torsional mode of the wing, the second bending mode of the wing, the in-plane bending mode of the missile, the first bending mode of the missile, and the second torsional mode of the wing, respectively. Among the first six modes, the in-plane bending mode of the missile has little effect on the flutter characteristics of the aeroelastic system.

2.2. Unsteady Aerodynamic Model. Although the doublelattice method has been widely used to compute the unsteady aerodynamic loads in subsonic regime, it can not be used to predict the unsteady aerodynamic loads directly in the transonic regime because of the nonlinear flow characteristics. In this study, for the transonic flight parameter, an efficient methodology proposed by Chen et al. [18] was used to generate the unsteady aerodynamic influence coefficient matrices $\mathbf{Q}\left(k, M_{\infty}\right)$, which depended on the freestream Mach number $M_{\infty}$ and reduced frequency $k$. The methodology was based on a transonic equivalent strip method with steady pressure data provided by CFD. Figure 2(a) presents the 


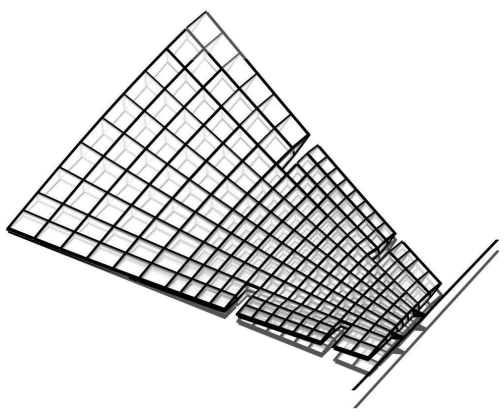

(a) Mode 1

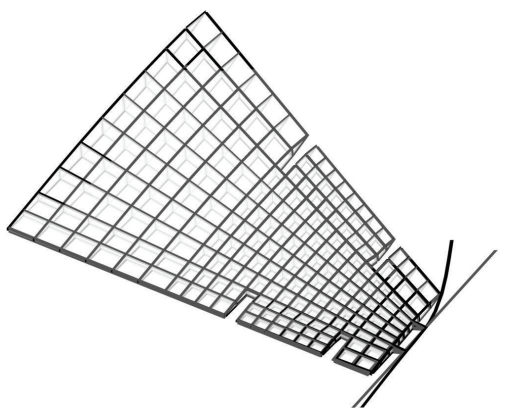

(d) Mode 4

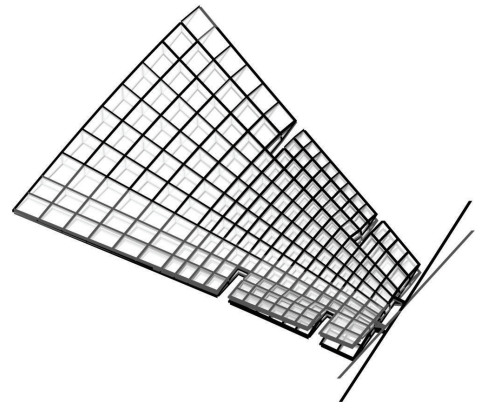

(b) Mode 2

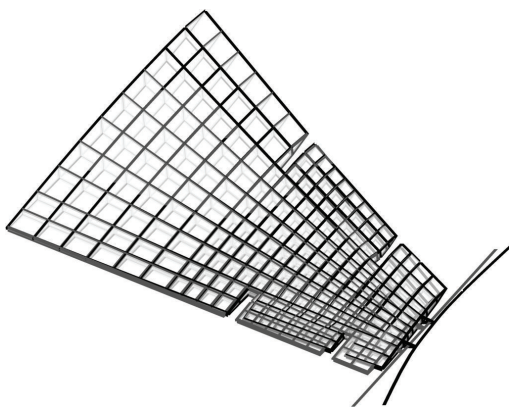

(e) Mode 5

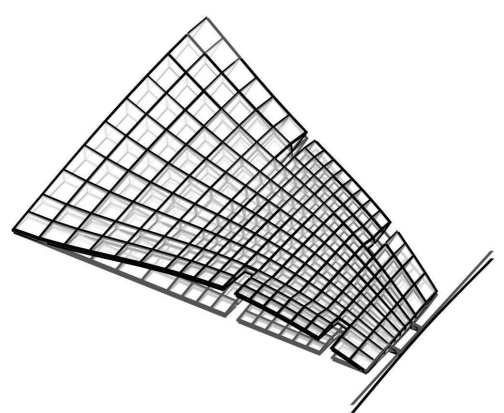

(c) Mode 3

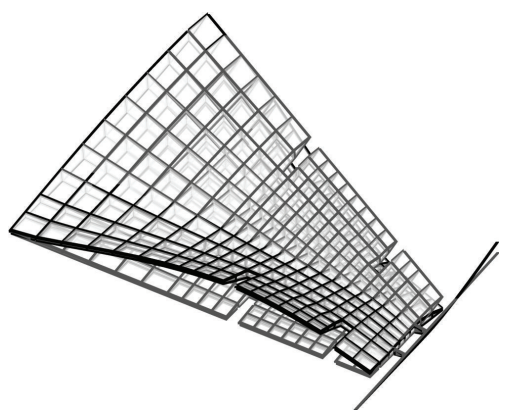

(f) Mode 6

FIGURE 1: Mode shapes of a typical fighter wing with a tip missile.

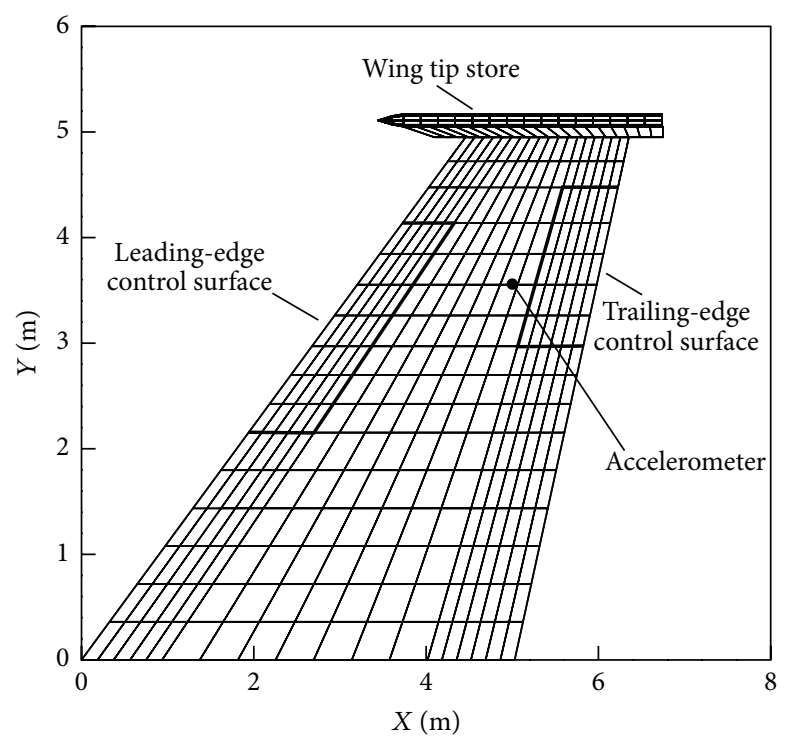

(a) Planform of the model and accelerometer location

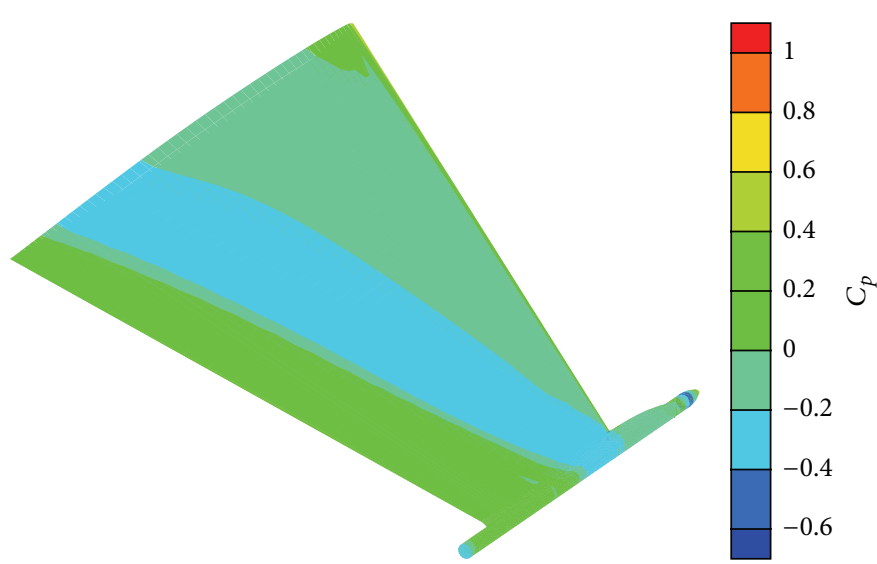

(b) Steady pressure coefficient at Mach number 0.95

FIGURE 2: Aerodynamic model of the typical fighter wing with a tip missile.

planform of the typical fighter wing with a tip missile and the aerodynamic grid used to compute the unsteady transonic aerodynamic influence coefficient matrices. The wing model was designed with symmetric NACA 65A004 airfoil and the steady pressure coefficient of the wing surface at zero angle of attack was computed for Mach number 0.95, as shown in Figure 2(b).
When the FEM and the unsteady aerodynamic influence coefficient matrices are available, the aeroelastic equation in frequency domain can be established as

$$
\begin{gathered}
{\left[\mathbf{M} s^{2}+\mathbf{D} s+\mathbf{K}-q_{\infty} \mathbf{Q}_{\mathbf{q}}\left(k, M_{\infty}\right)\right] \mathbf{q}} \\
=\left[-\mathbf{M}_{\mathbf{q} \delta} s^{2}+q_{\infty} \mathbf{Q}_{\boldsymbol{\delta}}\left(k, M_{\infty}\right)\right] \boldsymbol{\delta},
\end{gathered}
$$


where $\mathbf{M}, \mathbf{D}$, and $\mathbf{K}$ are the generalized mass, damping, and stiffness matrices and $\mathbf{q}$ is the vector of generalized displacements. In (1) $\mathbf{M}_{\mathbf{q} \delta}$ represents the inertia coupling between the control surfaces and the wing and $q_{\infty}$ represents the freestream dynamic pressure. The rigid deflections of control surfaces are represented by $\boldsymbol{\delta}$. In (1), $\mathbf{Q}_{\mathbf{q}}\left(k, M_{\infty}\right)$ and $\mathbf{Q}_{\delta}\left(k, M_{\infty}\right)$ represent the unsteady transonic aerodynamic influence coefficient matrices. Based on the rational function approximation proposed by Karpel and Strul [19], the unsteady aerodynamic influence coefficient matrices can be transformed into those in time domain. Now, (1) is transformed into the aeroelastic equation in state space as follows:

$$
\dot{\mathbf{x}}_{\mathrm{ae}}=\mathbf{A}_{\mathrm{ae}} \mathbf{x}_{\mathrm{ae}}+\mathbf{B}_{\mathrm{ae}} \mathbf{u}_{\mathrm{ae}},
$$

where

$$
\begin{aligned}
& \mathbf{x}_{\mathrm{ae}}=\left[\begin{array}{lll}
\mathbf{q} & \dot{\mathbf{q}} & \mathbf{x}_{a}
\end{array}\right]^{T}, \\
& \mathbf{u}_{\mathrm{ae}}=\left[\begin{array}{lll}
\boldsymbol{\delta} & \dot{\boldsymbol{\delta}} & \ddot{\boldsymbol{\delta}}
\end{array}\right]^{T}, \\
& \mathrm{~A}_{\mathrm{ae}} \\
& =\left[\begin{array}{ccc}
\mathbf{0} & \mathbf{I} & \mathbf{0} \\
-\overline{\mathbf{M}}^{-1}\left(\mathbf{K}-q_{\infty} \mathbf{A}_{\mathbf{q} 0}\right) & -\overline{\mathbf{M}}^{-1}\left(\mathbf{D}-q_{\infty} \frac{b}{V_{\infty}} \mathbf{A}_{\mathbf{q 1}}\right) & q_{\infty} \overline{\mathbf{M}}^{-1} \mathbf{D}_{s} \\
\mathbf{0} & \mathbf{E}_{\mathbf{q} s} & \frac{b}{V_{\infty}} \mathbf{R}_{s}
\end{array}\right], \\
& \mathbf{B}_{\text {ae }}
\end{aligned}
$$

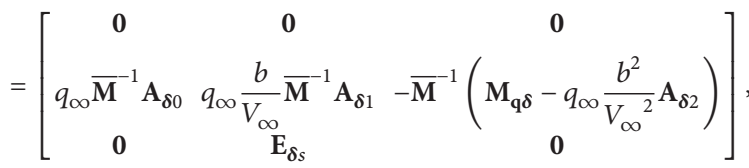

$$
\begin{aligned}
& \overline{\mathbf{M}}=\mathbf{M}-q_{\infty} \frac{b^{2}}{V_{\infty}^{2}} \mathbf{A}_{\mathbf{q} 2} \text {. }
\end{aligned}
$$

The output equations of the accelerometers attached to the wing can be given as

$$
\mathbf{y}_{\mathrm{ae}}=\mathbf{C}_{\mathrm{ae}} \mathbf{x}_{\mathrm{ae}}+\mathbf{D}_{\mathrm{ae}} \mathbf{u}_{\mathrm{ae}}
$$

where

$$
\begin{aligned}
& \mathbf{C}_{\mathrm{ae}} \\
& =\boldsymbol{\Phi}_{\mathrm{acc}} \overline{\mathbf{M}}^{-1}\left[-\left(\mathbf{K}-q_{\infty} \mathbf{A}_{\mathbf{q} 0}\right)-\left(\mathbf{D}-q_{\infty} \frac{b}{V_{\infty}} \mathbf{A}_{\mathbf{q} 1}\right) q_{\infty} \mathbf{D}_{s}\right], \\
& \mathbf{D}_{\mathrm{ae}} \\
& =\boldsymbol{\Phi}_{\mathrm{acc}} \overline{\mathbf{M}}^{-1}\left[q_{\infty} \mathbf{A}_{\boldsymbol{\delta} 0} q_{\infty} \frac{b}{V_{\infty}} \mathbf{A}_{\boldsymbol{\delta} 1}-\left(\mathbf{M}_{\mathbf{q} \boldsymbol{\delta}}-q_{\infty} \frac{b^{2}}{V_{\infty}^{2}} \mathbf{A}_{\boldsymbol{\delta} 2}\right)\right] .
\end{aligned}
$$

$\Phi_{\text {acc }}$ in (5) is the modal displacement matrix at the acceleration sensor location.

2.3. Actuator Model. The dynamics of an actuator for a control surface can be represented in frequency domain as

$$
\frac{\delta_{i}(s)}{u_{a c i}(s)}=\frac{a_{3, i}}{s^{3}+a_{1, i} s^{2}+a_{2, i} s+a_{3, i}},
$$

where $\delta_{i}$ is the control surface deflection and $u_{a c i}$ is the control command of the $i$ th control surface. The corresponding equation of (6) in state space is given by

$$
\dot{\mathbf{x}}_{i}=\left[\begin{array}{ccc}
0 & 1 & 0 \\
0 & 0 & 1 \\
-a_{3, i} & -a_{2, i} & a_{1, i}
\end{array}\right] \mathbf{x}_{i}+\left[\begin{array}{c}
0 \\
0 \\
a_{3, i}
\end{array}\right] u_{a c i}, \quad \mathbf{x}_{i}=\left[\begin{array}{c}
\delta_{i} \\
\dot{\delta}_{i} \\
\ddot{\delta}_{i}
\end{array}\right] .
$$

For multiple control surfaces, the dynamic equations of all actuators in the state space can be arranged in a corresponding order as shown by (7). Thus, the assembled dynamic equations of all actuators in the state space are in the following form:

$$
\dot{\mathbf{x}}_{\mathrm{ac}}=\mathbf{A}_{\mathrm{ac}} \mathbf{x}_{\mathrm{ac}}+\mathbf{B}_{\mathrm{ac}} \mathbf{u}_{\mathrm{ac}}, \quad \mathbf{x}_{\mathrm{ac}}=\left[\begin{array}{lll}
\boldsymbol{\delta} & \dot{\boldsymbol{\delta}} & \ddot{\boldsymbol{\delta}}
\end{array}\right]^{T} .
$$

The final open-loop aeroservoelastic model in the state space is a combination of the aeroelastic model and the actuator model as follows:

$$
\begin{aligned}
& \dot{\mathbf{x}}_{p}=\mathbf{A}_{p} \mathbf{x}_{p}+\mathbf{B}_{p} \mathbf{u}_{p}, \\
& \mathbf{y}_{p}=\mathbf{C}_{p} \mathbf{x}_{p},
\end{aligned}
$$

where

$$
\begin{aligned}
& \mathbf{A}_{p}=\left[\begin{array}{cc}
\mathbf{A}_{\mathrm{ae}} & \mathbf{B}_{\mathrm{ae}} \\
\mathbf{0} & \mathbf{A}_{\mathrm{ac}}
\end{array}\right], \\
& \mathbf{B}_{p}=\left[\begin{array}{c}
\mathbf{0} \\
\mathbf{B}_{\mathrm{ac}}
\end{array}\right], \\
& \mathbf{x}_{p}=\left[\begin{array}{l}
\mathbf{x}_{\mathrm{ae}} \\
\mathbf{x}_{\mathrm{ac}}
\end{array}\right], \\
& \mathbf{u}_{p}=\mathbf{u}_{\mathrm{ac}}, \\
& \mathbf{C}_{p}=\left[\begin{array}{ll}
\mathbf{C}_{\mathrm{ae}} & \mathbf{D}_{\mathrm{ae}}
\end{array}\right] .
\end{aligned}
$$

\section{Control Methodology}

According to the theory of RNNs in Bernelli-Zazzera and Lo-Rizzo [20], an adaptive controller with a recurrent neural network structure, as shown in Figure 3, can be constructed to extend the flutter boundary of the aeroelastic systems over a wide range of flight conditions.

This controller consists of two networks, that is, the identification network and the control network as shown in Figure 4. At time instant $k$, the identification network is used to predict the acceleration signal $\mathbf{y}^{p}(k+1)$ of time instant $k+1$ such that a predictive control can be implemented for the aeroelastic system. The prediction of acceleration signal $\mathbf{y}^{p}(k+1)$ generated by the identification network is used as part of the input variables for the control network. The output of the control network is the desired control command $\mathbf{u}^{c}(k+$ 1) to stabilize the aeroelastic system. The synaptic weights of the identification network and the control network are updated online according to a real-time recurrent learning (RTRL) algorithm proposed by Williams and Zipser [21]. In this section, the main parts of the identification network and the control network are presented. 


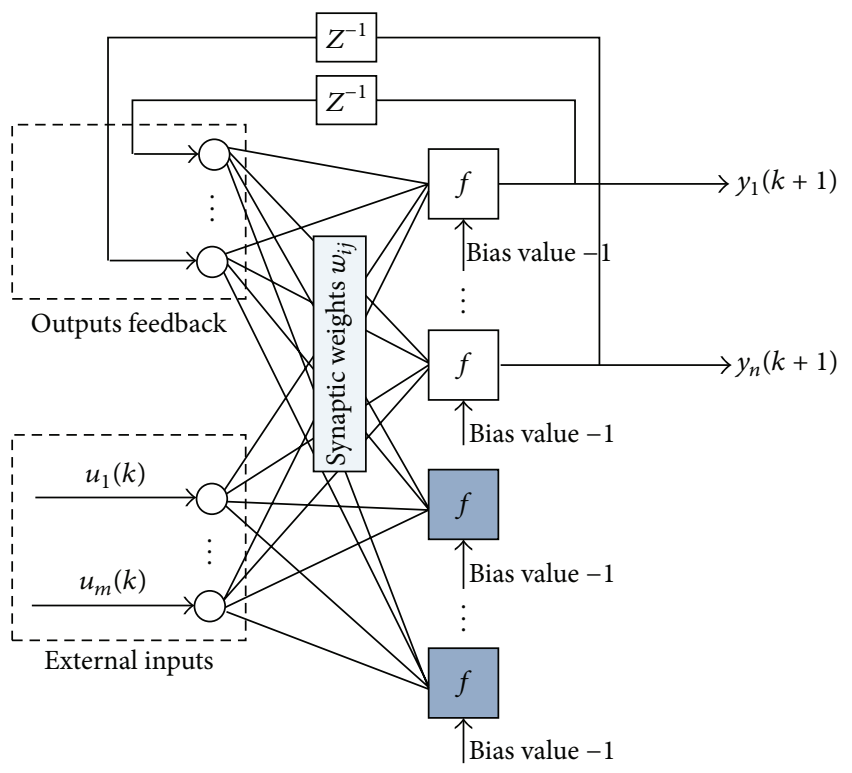

$f$ Visible neurons

$f$ Hidden neurons

FIGURE 3: Recurrent neural network structure.

3.1. Identification Network. In this work, the identification network is used to make one-step ahead prediction of the acceleration signal measured by the accelerometers, which are attached to particular points of the wing. As shown in Figure 4 , the inputs to the identification network contain the accelerometer measurement $y^{m}(k)$, the control command $\mathbf{u}^{c}(k)$, and the feedback signals from all neurons of the identification network. Hence,

$$
\mathbf{u}^{\mathrm{Id}}(k)=\left[\begin{array}{lllll}
\mathbf{y}^{\mathrm{Id}}(k) & \mathbf{y}^{m}(k) & \mathbf{u}^{c}(k) & -1
\end{array}\right]^{T},
$$

where $y^{\text {Id }}(k)$ are the feedback signals from all neurons of the identification network. Then, the outputs from all neurons of the identification network can be evaluated as

$$
\begin{aligned}
v_{j}^{\mathrm{Id}}(k) & =\sum_{i=1}^{N_{\mathrm{Id}}} w_{j i}^{\mathrm{Id}}(k) u_{i}^{\mathrm{Id}}(k), \\
y_{j}^{\mathrm{Id}}(k+1) & =\alpha \tanh \left(\beta v_{j}^{\mathrm{Id}}(k)\right),
\end{aligned}
$$

where $w_{j i}^{\mathrm{Id}}(k)$ is a synaptic weight of the identification network. The acceleration predictions $\mathbf{y}^{p}(k+1)$ are the outputs from all the visible neurons of the identification network. The identification network is trained online according to the RTRL algorithm to minimize the quadratic identification error $E^{\text {Id }}$ defined as

$$
E^{\mathrm{Id}}=\frac{1}{2} \sum_{i=1}^{N^{m}}\left(y_{i}^{m}(k)-y_{i}^{p}(k)\right)^{2},
$$

where $N^{m}$ is the number of accelerometers mounted on the wing. Through the RTRL algorithm, the synaptic weights are updated online as follows:

$$
w_{j i}^{\mathrm{Id}}(k+1)=w_{j i}^{\mathrm{Id}}(k)-\eta^{\mathrm{Id}} \frac{\partial E^{\mathrm{Id}}(k)}{\partial w_{j i}^{\mathrm{Id}}(k)},
$$

where $\eta^{\text {Id }}$ is the learning rate of the identification network. As a high learning rate may stabilize the training algorithm and a low value will limit the capability of the identification network to quickly adapt the network to system variations, the learning rate needs to be chosen as a compromise. Because there is no strict theory about how to choose the appropriate learning rate, the value has to be tuned through extensive numerical tests.

3.2. Control Network. Because the aeroelastic system to be controlled in this study is a nonminimum phase system, the control network is based on a pseudo inversion technique as shown in Bernelli-Zazzera et al. [16] to obtain the desired control command signals $\mathbf{u}^{c}(k+1)$. The inputs to the control network contain the acceleration predictions $y^{p}(k+1)$, the control command $\mathbf{u}^{c}(k)$, and the feedback signals from all neurons of the control network. Hence, one has

$$
\mathbf{u}^{\text {Con }}(k)=\left[\begin{array}{llll}
\mathbf{y}^{\text {Con }}(k) & \mathbf{y}^{p}(k+1) & \mathbf{u}^{c}(k) & -1
\end{array}\right]^{T},
$$

where $\mathrm{y}^{\mathrm{Con}}(k)$ is the feedback signals from all neurons of the control network. Similar to the identification network, the outputs from all neurons of the control network are evaluated as

$$
\begin{aligned}
v_{j}^{\text {Con }}(k) & =\sum_{i=1}^{N_{\text {Con }}} w_{j i}^{\text {Con }}(k) u_{i}^{\text {Con }}(k), \\
y_{j}^{\text {Con }}(k+1) & =\alpha \tanh \left(\beta v_{j}^{\text {Con }}(k)\right),
\end{aligned}
$$

where $w_{j i}^{\text {Con }}(k)$ is a synaptic weight of the control network. The desired control command signals $\mathbf{u}^{c}(k+1)$ are the outputs from all the visible neurons of the control network. The cost function to minimize for the pseudo inversion technique is

$$
\begin{aligned}
E^{\text {Con }}= & \frac{1}{2} \sum_{i=1}^{N^{m}}\left(y_{i}^{m}(k+1)-y_{i}^{p}(k+1)\right)^{2} \\
& +\frac{1}{2} \gamma \sum_{i=1}^{N^{c}}\left(u_{i}^{c}(k)\right)^{2},
\end{aligned}
$$

where a penalization term due to the control action is introduced to limit the control effort and $\gamma$ is the weight of the penalization term. For the nonminimum phase system, the penalization term is necessary to make the control network stable as shown in Isermann et al. [22]. The control network is trained online according to RTRL algorithm. The synaptic weights are updated to minimize the cost function of the control network as given by

$$
w_{j i}^{\text {Con }}(k+1)=w_{j i}^{\text {Con }}(k)-\eta^{\text {Con }} \frac{\partial E^{\text {Con }}(k)}{\partial w_{j i}^{\text {Con }}(k)},
$$




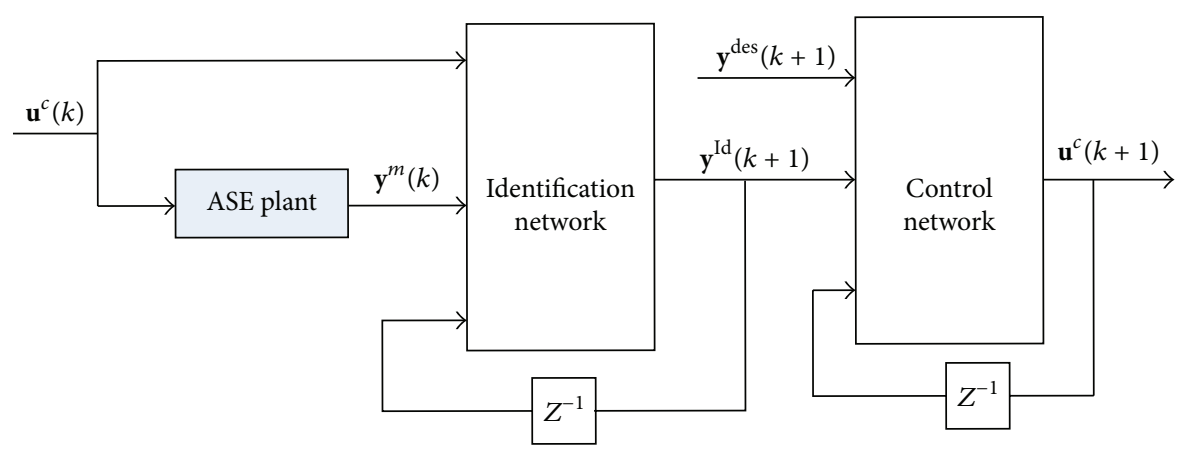

FIGURE 4: Block diagram of the active flutter suppression system.
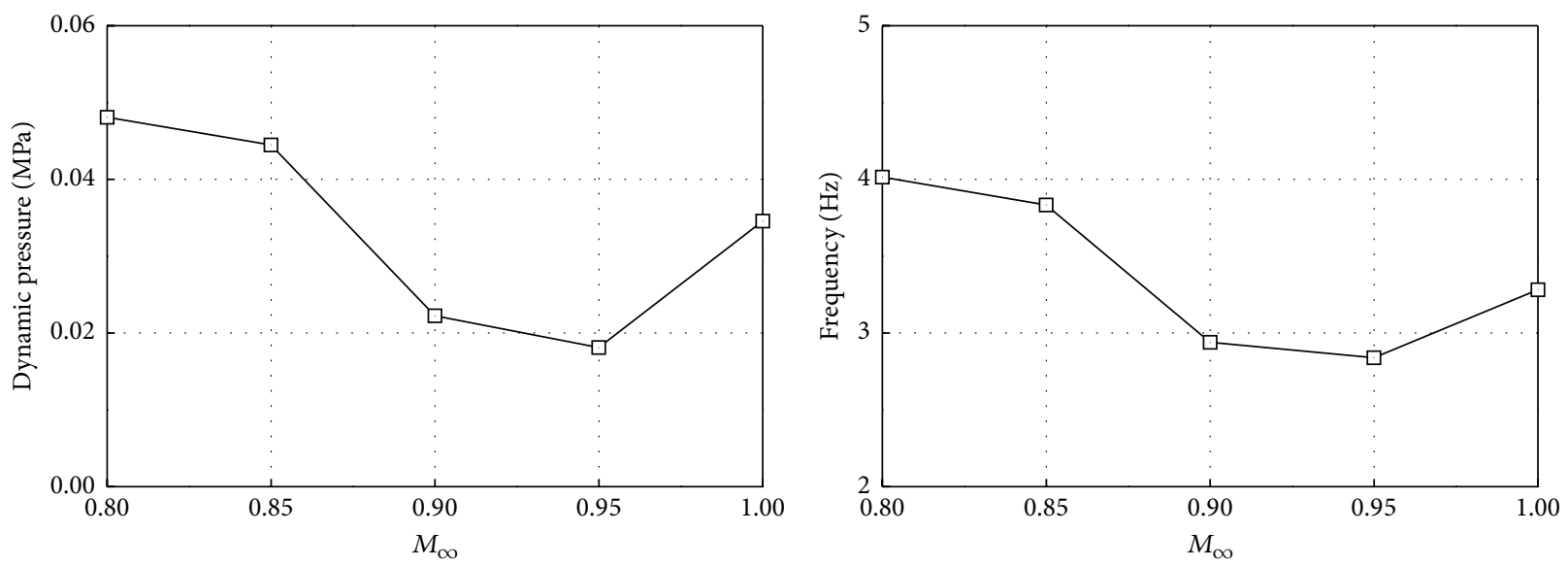

FIGURE 5: Flutter boundary of the wing model.

where $\eta^{\text {Con }}$ is the learning rate of the control network. The value of the learning rate also has to be tuned through extensive numerical tests to maximize the performance of the controller.

\section{Numerical Simulations}

4.1. Open-Loop Aeroservoelastic Analyses. No-matched point flutter analysis was performed first for the fighter wing model described in Section 2. Figure 5 presents the flutter boundary of the open-loop ASE system, with respect to the Mach numbers $0.80,0.85,0.90,0.95$, and 1.0 , respectively. The figure clearly shows a drop of the flutter boundary in the transonic regime, which limits the performance of the wing.

4.2. Closed-Loop Aeroservoelastic Analyses. Figure 2(a) presents the location of accelerometer used for flutter stabilization. The trailing-edge control surface was used to suppress possible flutters. According to the theory of RNNs based controller shown in Section 3, the single-input/single-output (SISO) flutter suppression system was synthesized to improve the performance of the typical wing with a tip missile.

At first, the sample frequency was set to be $200 \mathrm{~Hz}$ as a tradeoff between the computational power of computer in a real flight and the following ability of control system. Extensive numerical simulations were performed to tune the
TABle 2: Parameters of the identification network and the control network.

\begin{tabular}{lcc}
\hline & Identification network & Control network \\
\hline Number of neurons & 6 & 6 \\
Learning rate & 0.3 & 0.2 \\
Weight of penalty term & - & 0.2 \\
\hline
\end{tabular}

parameters of networks, such as number of neurons, the learning rate, and the weight of the penalization term. The parameters of the identification network and the control network were determined and presented in Table 2. The synaptic weights of networks were initialized with the weights determined during numerical simulations at Mach number of 0.80 and postflutter dynamic pressure of $51.8 \mathrm{kPa}$.

To evaluate the capability of the identification network, the control command of the trailing-edge control surface was selected as white noise. Figure 6(a) shows the control command of the trailing-edge control surface at Mach number of 1.00 and dynamic pressure of $37.6 \mathrm{kPa}$. Figure $6(\mathrm{~b})$ shows the corresponding acceleration signal measured and the signal predicted by the identification network. As shown in Figure 6, the synaptic weights are able to generate a fairly good prediction after only $5 \mathrm{~s}$, which shows that the identification network can follow the aeroelastic system quickly. 


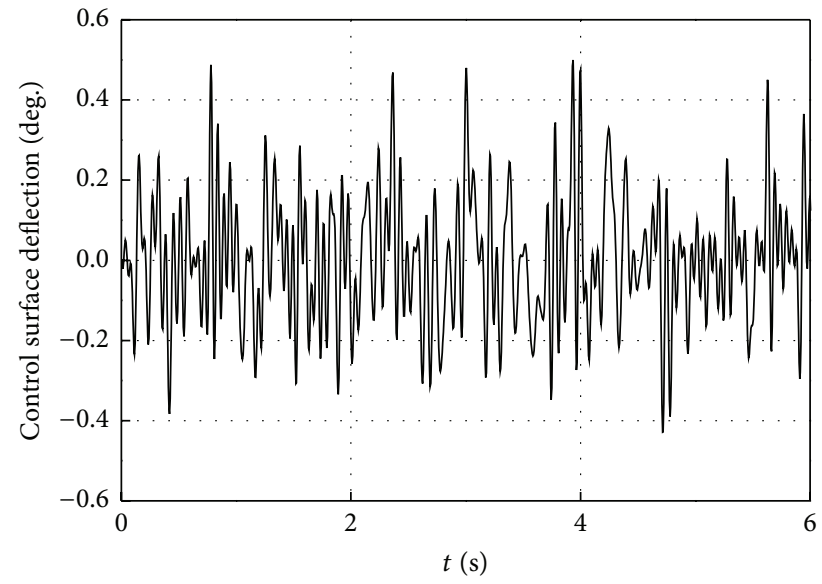

(a) Deflection command of trailing-edge control surface

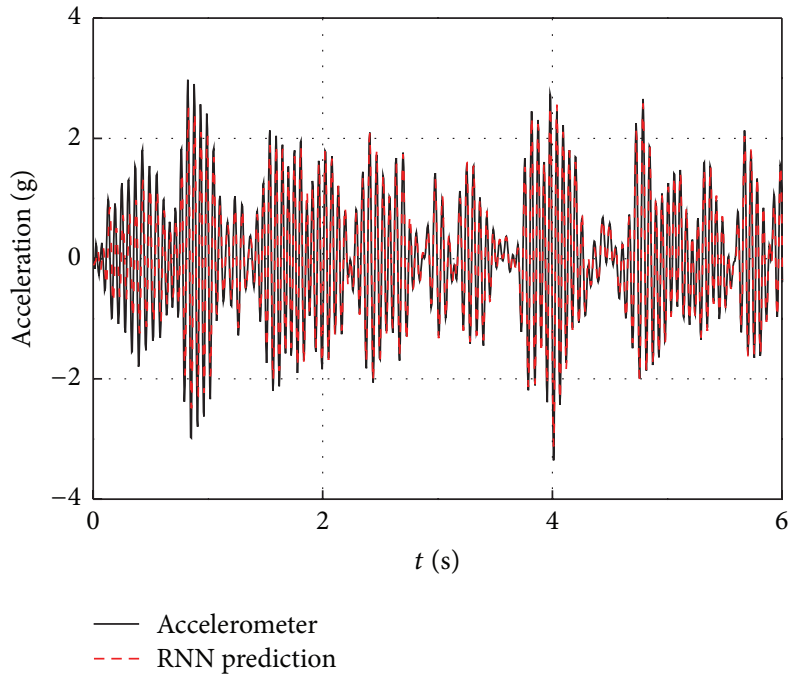

(b) Comparison of acceleration signals

FIGURE 6: Prediction capability of the identification network.

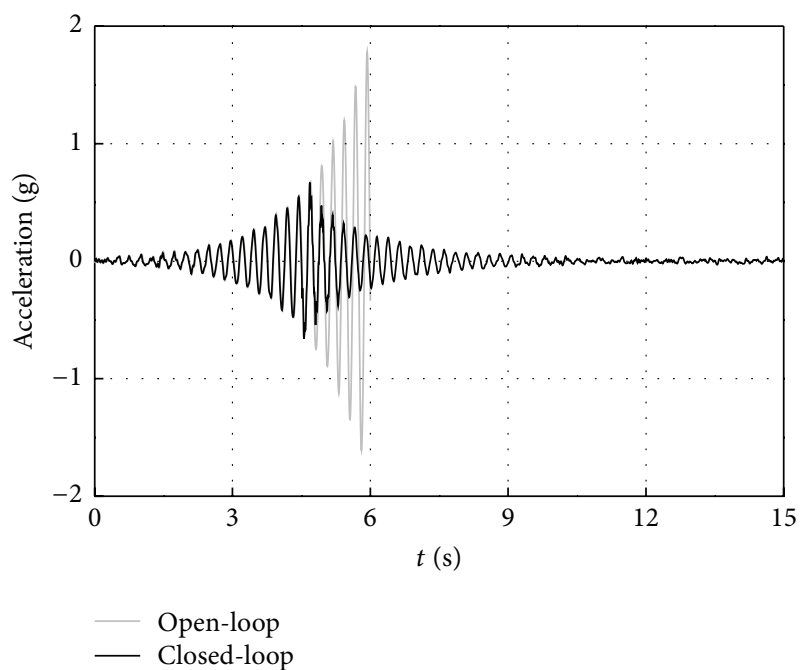

(a) Open- and closed-loop accelerations

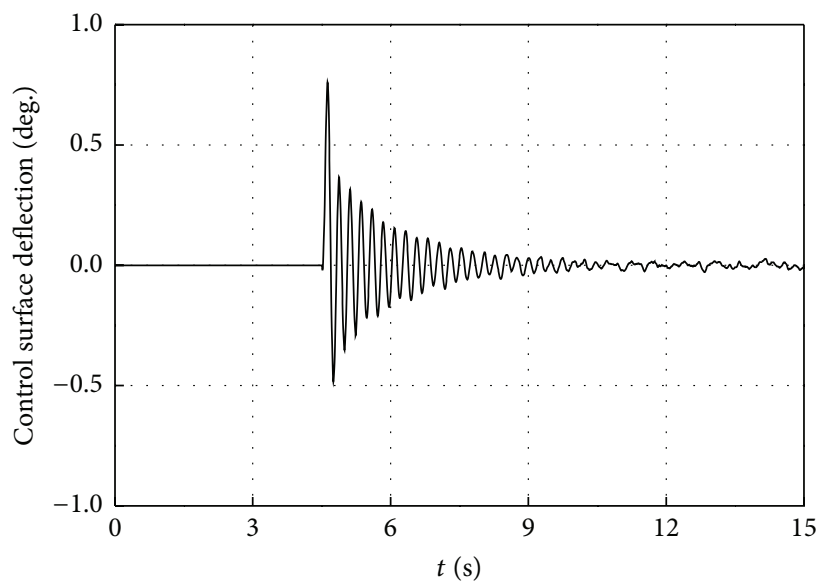

(b) Control command of trailing-edge control surface

Figure 7: Control effectiveness at a Mach number of 0.80 .

To testify the robustness of the proposed adaptive controller for flutter suppression, the accelerometer signals were assumed to contain the white noise with intensity of $0.05 \mathrm{~g}$. Figure 7 (a) presents the accelerations of open- and closed-loop systems at Mach number of 0.80 and under postflutter dynamic pressure of $51.8 \mathrm{kPa}$. Figure $7(\mathrm{~b})$ gives the corresponding control command of the trailing-edge control surface. To test the adaptive ability of the controller at different flight conditions, Figure 8(a) presents the accelerations of open- and closed-loop systems at Mach number 0.90 and under a dynamic pressure $32.8 \mathrm{kPa}$. Figure $8(\mathrm{~b})$ presents the corresponding control command of the trailingedge control surface, too. Although the measured accelerations were assumed to be contaminated by noise, Figures
7 and 8 clearly showed that the flutter instability of the wing was effectively suppressed via the RNNs based controller.

Figure 9 presents the comparison of the dynamic pressures of open- and closed-loop flutters. Figure 10 shows the increase of dynamic pressure of flutter via the controller at different Mach numbers. There is a little increase in flutter boundary at Mach numbers of 0.80 and 0.85 as shown in Figures 9 and 10. The flutter boundary was extended significantly over the transonic dip where the performance of the fighter wing was limited, such as Mach numbers 0.90, 0.95 , and 1.0 .

Numerical results in this section showed that RNNs based controller could be used to stabilize the flutter of wing 


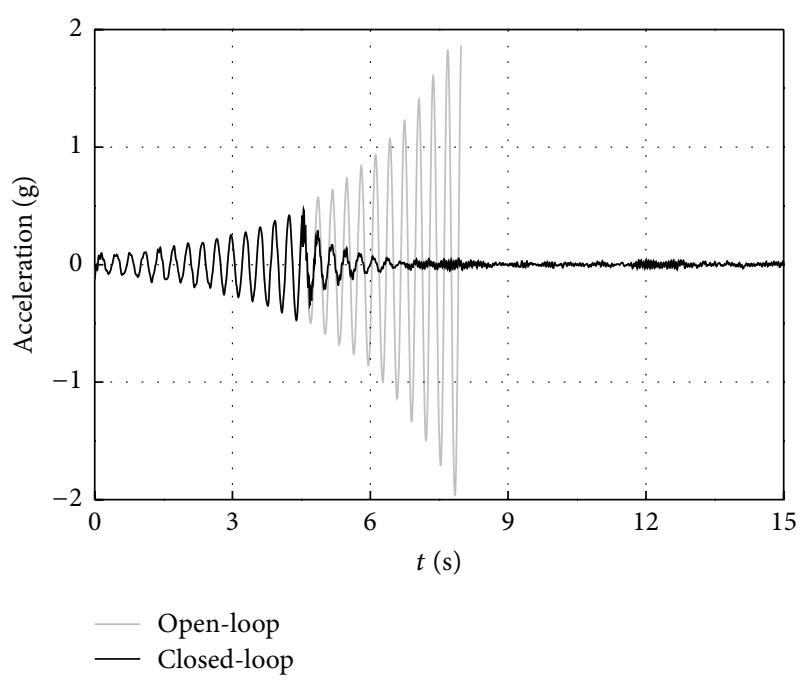

(a) Open- and closed-loop accelerations

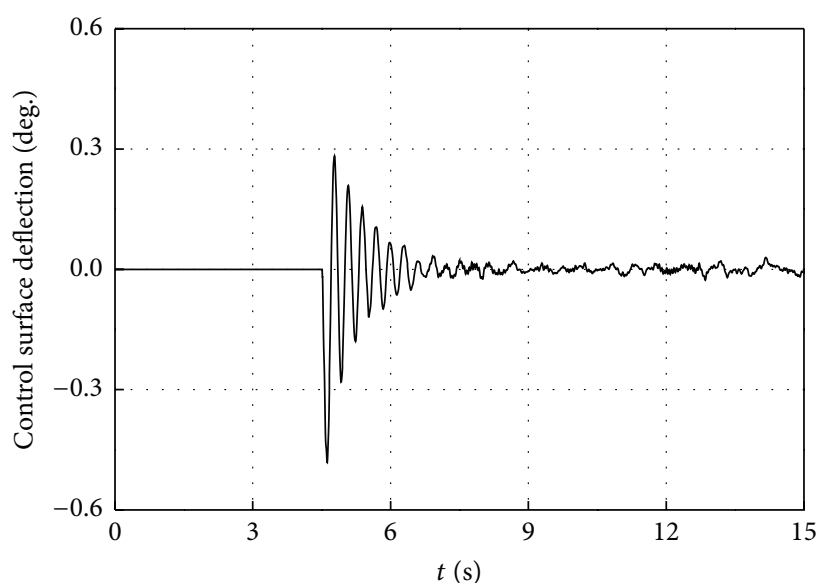

(b) Control command of trailing-edge control surface

FIGURE 8: Control effectiveness at a Mach number of 0.90.

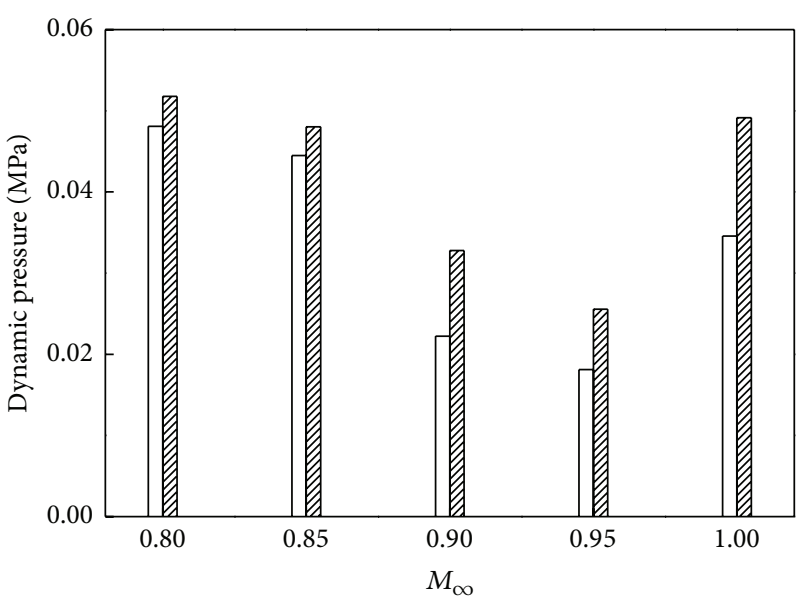

Open-loop flutter boundary WIIA Closed-loop flutter boundary

Figure 9: Open/closed-loop flutter boundary of the wing model.

structure over a wide range of Mach numbers. Hence, the proposed adaptive controller exhibited excellent robustness.

\section{Conclusions}

In this study, an adaptive flutter controller was synthesized via recurrent neural networks to suppress the instability of a wing structure over a wide transonic regime. The digital controller was verified by numerical simulations. Although the wing structure changed the stability features with the variation of flight conditions, the adaptive controller could automatically adjust itself to current flight condition by updating the synaptic weights of networks online via the real-time recurrent learning algorithm. An important advantage of the proposed controller was no requirement

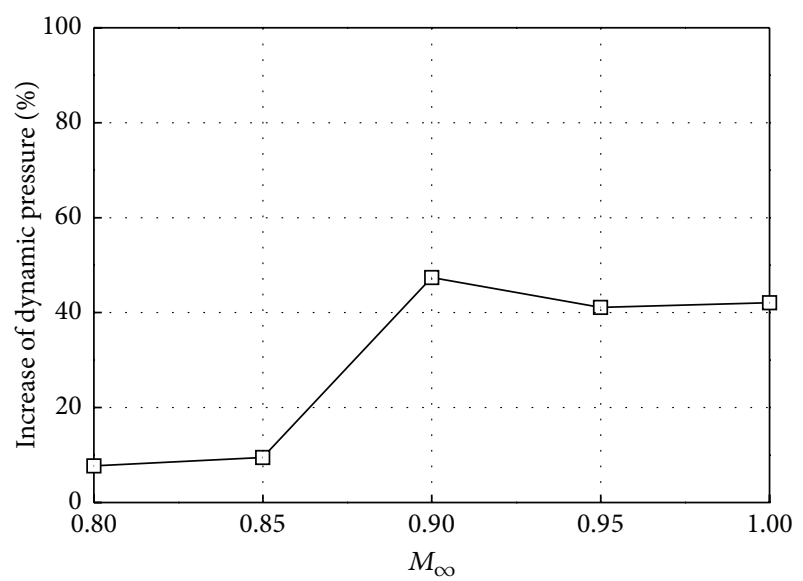

FIGURE 10: Increase of dynamic pressure of flutter via the controller.

for any information of the aeroelastic system except for the acceleration signals measured. The aeroservoelastic model of a typical fighter wing with a tip missile was established and a single-input/single-output controller was synthesized. The adaptive flutter controller was testified to successfully extend the flutter boundary of the model, especially in the transonic dip region where the performance of the model is limited. Although a three-dimensional wing model with a symmetric airfoil was studied in this study, the active flutter controller for the nonsymmetric airfoil wings can also be synthesized via the same approach. It should be noted, however, that the static aeroelastic effect has to be taken into consideration for the nonsymmetric airfoil wings.

\section{Competing Interests}

The authors declare that they have no competing interests. 


\section{Acknowledgments}

This work was supported by the National Natural Science Foundation of China under Grant 11472128.

\section{References}

[1] V. Mukhopadhyay, "Flutter suppression control law design and testing for the active flexible wing," Journal of Aircraft, vol. 32, no. 1, pp. 45-51, 1995.

[2] V. Mukhopadhyay, "Transonic flutter suppression control law design and wind-tunnel test results," Journal of Guidance, Control, and Dynamics, vol. 23, no. 5, pp. 930-937, 2000.

[3] Y. H. Zhao, "Flutter suppression of a high aspect-ratio wing with multiple control surfaces," Journal of Sound and Vibration, vol. 324, no. 3-5, pp. 490-513, 2009.

[4] B. Moulin, E. Ritz, J. R. Florance, M. D. Sanetrik, and W. A. Silva, "CFD-based classic and robust aeroservoelastic control for the supersonic semispan transport wind-tunnel model," in Proceedings of the 51st AIAA Atmospheric Flight Mechanics Conference, AIAA Paper 2010-7802, Toronto, Canada, August 2010.

[5] J. Zeng, S. L. Kukreja, and B. Moulin, "Experimental modelbased aeroelastic control for flutter suppression and gust-load alleviation," Journal of Guidance, Control, and Dynamics, vol. 35, no. 5, pp. 1377-1390, 2012.

[6] M. Andrighettoni and P. Mantegazza, "Multi-input/multioutput adaptive active flutter suppression for a wing model," Journal of Aircraft, vol. 35, no. 3, pp. 462-469, 1998.

[7] R. Zhang and S. N. Singh, "Adaptive output feedback control of an aeroelastic system with unstructured uncertainties," Journal of Guidance, Control, and Dynamics, vol. 24, no. 3, pp. 502-509, 2001.

[8] A. Behal, V. M. Rao, P. Marzocca, and M. Kamaludeen, "Adaptive control for a nonlinear wing section with multiple flaps," Journal of Guidance, Control, and Dynamics, vol. 29, no. 3, pp. 744-749, 2006.

[9] R. Huang, H. Hu, and Y. Zhao, "Single-input/single-output adaptive flutter suppression of a three-dimensional aeroelastic system," Journal of Guidance, Control, and Dynamics, vol. 35, no. 2, pp. 659-665, 2012.

[10] A. Mannarino and P. Mantegazza, "Multifidelity control of aeroelastic systems: an immersion and invariance approach," Journal of Guidance, Control, and Dynamics, vol. 37, no. 5, pp. 1568-1582, 2014.

[11] K. W. Lee and S. N. Singh, "Adaptive control of multi-input aeroelastic system with constrained inputs," Journal of Guidance, Control, and Dynamics, vol. 38, no. 12, pp. 2337-2350, 2015.

[12] C.-G. Pak, P. P. Friedmann, and E. Livne, "Digital adaptive flutter suppression and simulation using approximate transonic aerodynamics," JVC/Journal of Vibration and Control, vol. 1, no. 4, pp. 363-388, 1995.

[13] R. C. Scott and L. E. Pado, "Active control of wind-tunnel model aeroelastic response using neural networks," Journal of Guidance, Control, and Dynamics, vol. 23, no. 6, pp. 1100-1108, 2000.

[14] G. Chen, J. Sun, and Y.-M. Li, "Adaptive reduced-order-modelbased control-law design for active flutter suppression," Journal of Aircraft, vol. 49, no. 4, pp. 973-980, 2012.
[15] R. Huang, H. Li, H. Hu, and Y. Zhao, "Open/closed-loop aeroservoelastic predictions via nonlinear, reduced-order aerodynamic models," AIAA Journal, vol. 53, no. 7, pp. 1812-1824, 2015.

[16] F. Bernelli-Zazzera, P. Mantegazza, G. Mazzoni, and M. Rendina, "Active flutter suppression using recurrent neural networks," Journal of Guidance, Control, and Dynamics, vol. 23, no. 6, pp. 1030-1036, 2000.

[17] M. Mattaboni, G. Quaranta, and P. Mantegazza, "Active flutter suppression for a three-surface transport aircraft by recurrent neural networks," Journal of Guidance, Control, and Dynamics, vol. 32, no. 4, pp. 1295-1307, 2009.

[18] P. C. Chen, D. Sarhaddi, and D. D. Liu, "Transonicaerodynamic-influence-coefficient approach for aeroelastic and MDO applications," Journal of Aircraft, vol. 37, no. 1, pp. 85-94, 2000.

[19] M. Karpel and E. Strul, "Minimum-state unsteady aerodynamic approximations with flexible constraints," Journal of Aircraft, vol. 33, no. 6, pp. 1190-1196, 1996.

[20] F. Bernelli-Zazzera and V. Lo-Rizzo, "Adaptive control of space structures via recurrent neural networks," Dynamics and Control, vol. 9, no. 1, pp. 5-20, 1999.

[21] R. J. Williams and D. Zipser, "A learning algorithm for continually running fully recurrent neural networks," Neural Computation, vol. 1, no. 2, pp. 270-280, 1989.

[22] R. Isermann, D. Matko, and K. H. Lachmann, Adaptive Control Systems, Prentice-Hall, Hemel Hempstead, UK, 1992. 


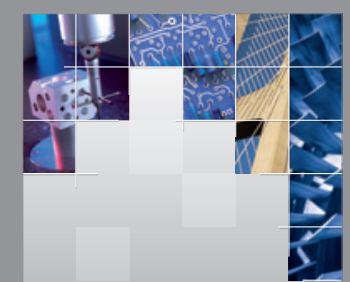

\section{Enfincering}
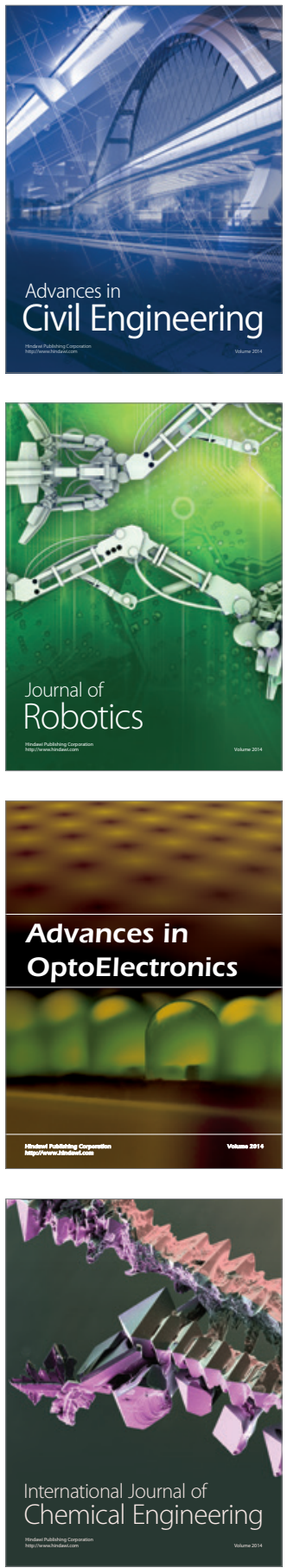

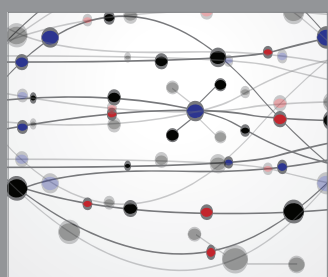

The Scientific World Journal

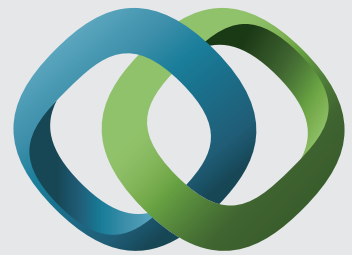

\section{Hindawi}

Submit your manuscripts at

http://www.hindawi.com
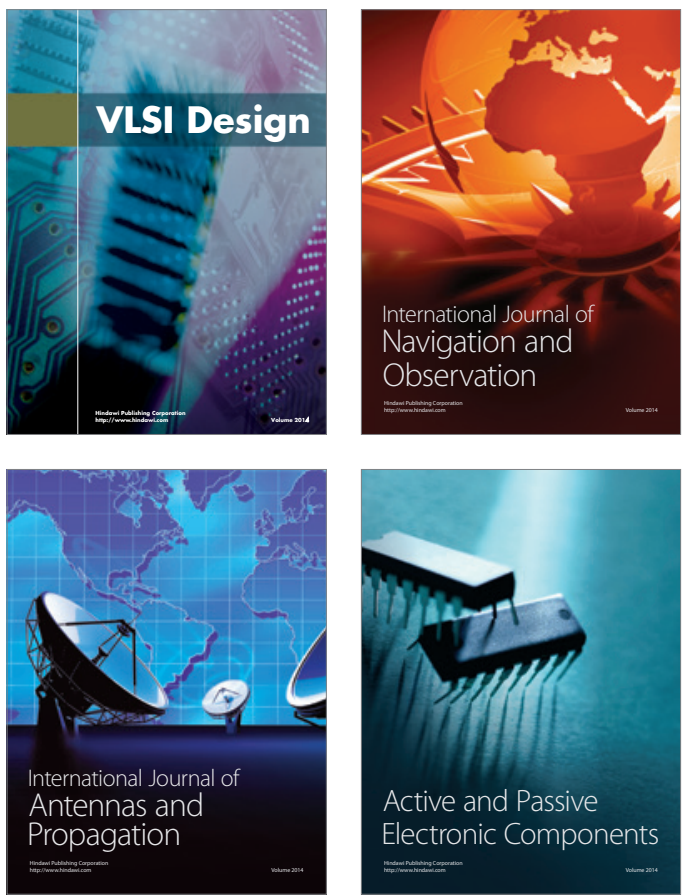
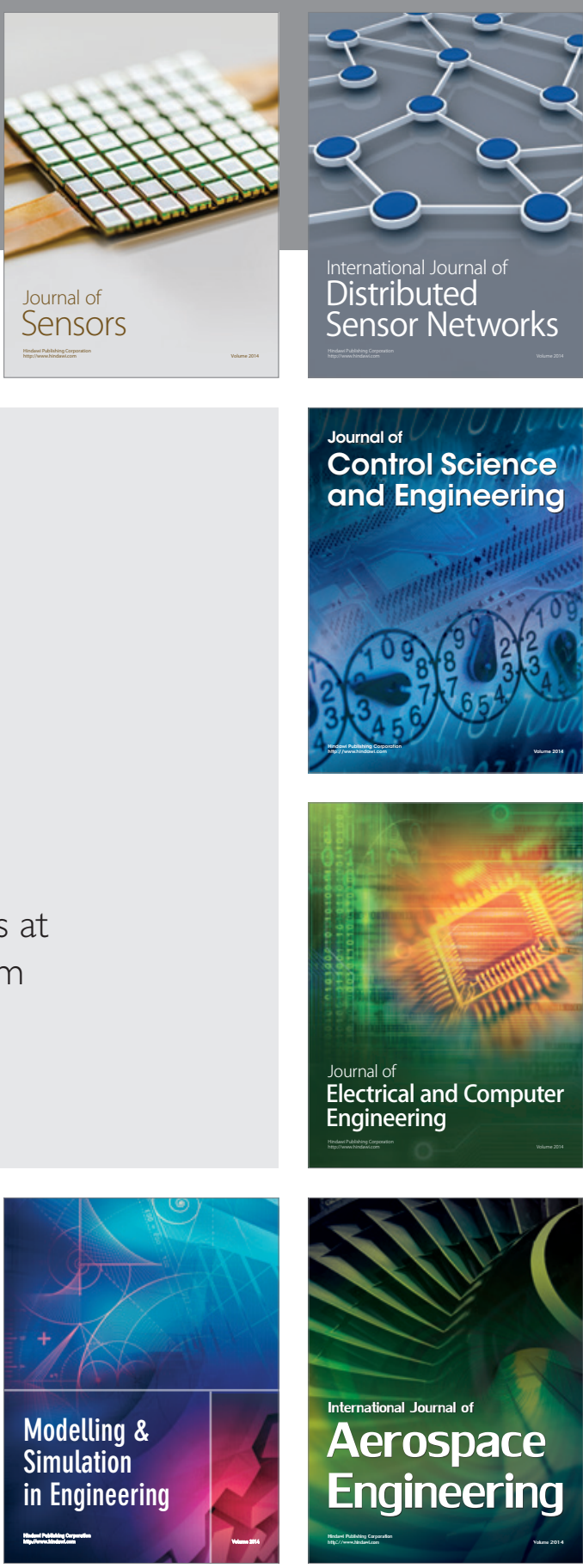

International Journal of

Distributed

Sensor Networks

Journal of

Control Science

and Engineering
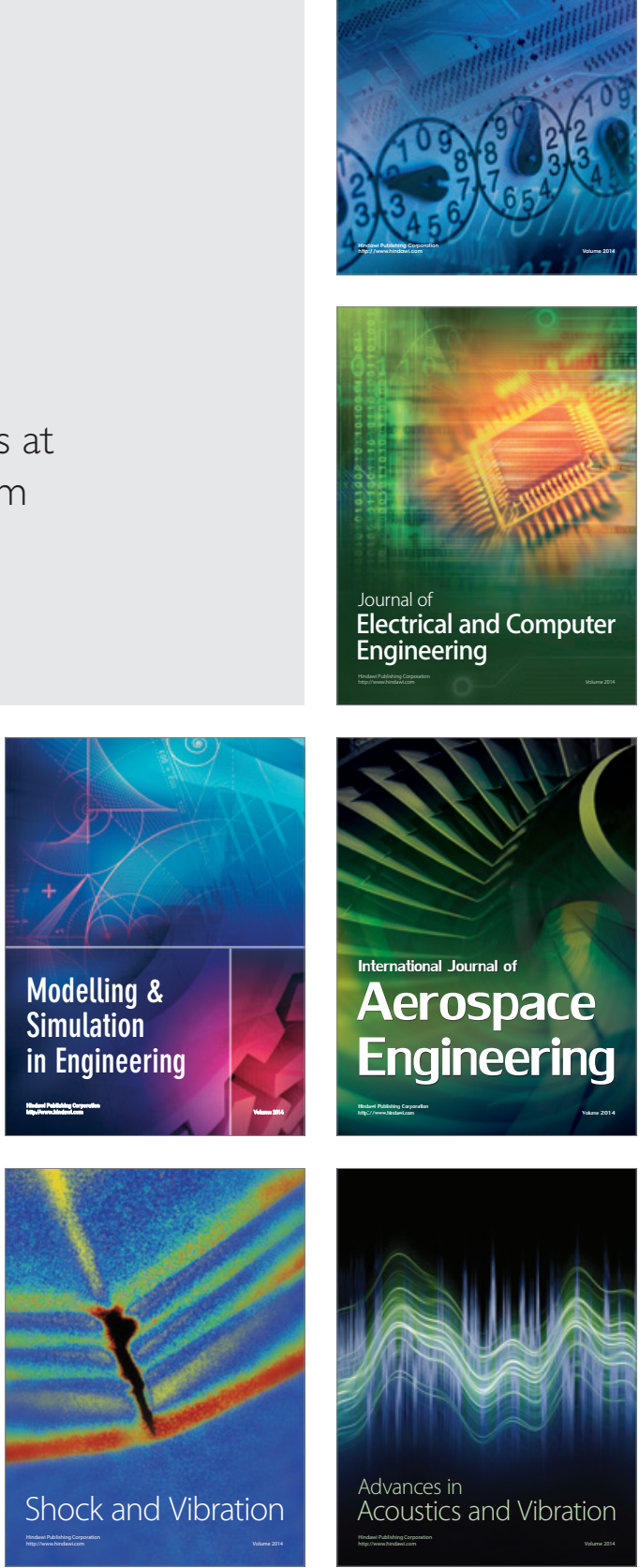